\title{
Paclitaxel for relapsed small-cell lung cancer patients with idiopathic interstitial pneumonias
}

\author{
ATSURO SAIJO, MASAKI HANIBUCHI, HIROKAZU OGINO, KENJI OTSUKA, \\ HISATSUGU GOTO, HIROSHI NOKIHARA and YASUHIKO NISHIOKA
}

\begin{abstract}
Department of Respiratory Medicine and Rheumatology, Graduate School of Biomedical Sciences, Tokushima University, Tokushima, Tokushima 770-8503, Japan
\end{abstract}

Received December 20, 2018; Accepted March 13, 2019

DOI: $10.3892 / \mathrm{mco} .2019 .1828$

\begin{abstract}
Although first-line chemotherapy is highly sensitive against small-cell lung cancer (SCLC), most patients subsequently experience disease progression. Topotecan is the standard therapy for sensitive-relapsed SCLC patients, and subgroup analysis of a randomized phase III trial suggests that amrubicin is effective for refractory-relapsed SCLC. However, because of the lack of the evidence based on clinical trials, the effectiveness of systemic chemotherapy for relapsed SCLC patients with idiopathic interstitial pneumonias (IIPs) is unclear. In the presentstudy, 17 relapsed SCLC patients with IIPs who received a paclitaxel (PTX)-containing regimen as a second-line chemotherapy were retrospectively reviewed. The overall response rate and the disease control rate of the PTX-containing regimens were 29.4 and $47.1 \%$, respectively. The median progression-free survival and the overall survival of the regimens were 2.7 months [95\% confidence interval (CI), 1.6-3.6 months] and 3.6 months (95\% CI, 2.3-14.0 months), respectively. Grade 3-4 neutropenia and febrile neutropenia occurred in $12(70.6 \%)$ and $2(11.8 \%)$ patients, respectively. During the treatment period, acute exacerbation (AE) of IIPs was observed in five patients (29.4\%). Treatment-associated fatality was observed in 1 patient with febrile neutropenia and in 1 patient with AE of IIPs. PTX had promising anti-tumor activity against refractory-relapsed SCLC with IIPs. However, the survival benefit of the treatment was limited because of the high incidence of AE of IIPs and treatment-related death.
\end{abstract}

Correspondence to: Professor Yasuhiko Nishioka, Department of Respiratory Medicine and Rheumatology, Graduate School of Biomedical Sciences, Tokushima University, 3-18-15 Kuramoto-cho, Tokushima, Tokushima 770-8503, Japan

E-mail: yasuhiko@tokushima-u.ac.jp

Key words: small-cell lung cancer, idiopathic interstitial pneumonias, acute exacerbation of idiopathic interstitial pneumonia, paclitaxel, second-line chemotherapy

\section{Introduction}

Small-cell lung cancer (SCLC) is a high-grade neuroendocrine lung cancer and comprises about $15 \%$ of all lung cancers (1). SCLC is characterized by rapid growth and early metastasis to distant organs, and most patients are diagnosed with extensive disease. Systemic chemotherapy is the standard treatment, and the response rate to first-line platinum-based chemotherapy is high. However, most patients experience disease progression while on or after first-line chemotherapy (2). The response of second-line chemotherapy depends on the interval time from the last day of first-line chemotherapy to the day of confirmed relapse: Sensitive disease (the interval $>90$ days) has an overall response rate (ORR) of approximately $25 \%$ and refractory disease (the interval $<90$ days) has an ORR of approximately $10 \%(3,4)$. Topotecan is safe and effective for the sensitive disease $(5,6)$. Subgroup analysis of a phase III trial suggested the effectiveness of amrubicin for patients with the refractory disease (7). Furthermore, a recent study demonstrated the antitumor activity with durable response of immune-checkpoint inhibitors, nivolumab monotherapy and nivolumab plus ipilimumab in patients with both sensitive and refractory disease (8). However, patients with severe complications including idiopathic interstitial pneumonias (IIPs) were excluded from these clinical trials, and therefore the survival benefit of chemotherapy for SCLC patients with IIPs is unclear.

IIPs are a common comorbidity of lung cancer and affect 5.8\% of surgically-resected lung cancer patients (9). Among patients with IIPs, the prevalence of lung cancer at the diagnosis of IIPs was 6-17\% (10). The presence of IIPs is an obstacle to systemic chemotherapy because of the risk of death and the deterioration of quality of life attributed to acute exacerbation (AE) of IIPs (11). A combination of platinum agents, cisplatin or carboplatin, plus etoposide is the standard treatment regimen for chemotherapy-naïve SCLC patients with extensive disease $(2,3)$. The safety and efficacy of these regimens for SCLC patients with IIPs or interstitial lung disease were evaluated in two retrospective studies and one prospective study in Japan. AE related to platinum agents plus etoposide was observed in $5.9 \%$ (1/17) of patients with IIPs and $1.9 \%(1 / 52)$ of patients with preexisting interstitial 
lung disease $(12,13)$. The ORRs, the median progression-free survival (PFS), and the median survival time (MST) of the combination of platinum agents plus etoposide for SCLC patients with IIPs were 63.6-88.2\%, 4.5-5.5 months, and 7.0-9.4 months, respectively (12-14). Compared to the MST of SCLC patients who received only supportive care, which is 2-3 months (15), these results reveal the benefit of first-line chemotherapy for SCLC patients with IIPs. In the second-line setting, topotecan-induced AE occurred in 20.0-23.8\% of SCLC patients with interstitial lung disease $(16,17)$. Two retrospective studies reported that the incidences of amrubicin-induced AE were 10\% (1/10 patients) and $17.6 \%$ (3/17 patients) in lung cancer patients with preexisting interstitial lung disease $(13,18)$. Thus, the standard chemotherapy regimens for relapsed SCLC may be ineligible for patients with IIPs because of the high incidence of AE.

The efficacy of single agent paclitaxel (PTX) for previously treated SCLC patients was assessed in two phase II studies, and the ORRs of PTX were 23.8 and $29.2 \%(19,20)$. PTX in combination with carboplatin is a candidate regimen for advanced non-small cell lung cancer (NSCLC) patients with IIPs in terms of the low incidence of AE (21). Furthermore, a retrospective study suggested the safety and effectiveness of second-line chemotherapy including PTX in relapsed SCLC patients with IIPs (16). Thus, these results suggested that PTX may be a treatment option for relapsed SCLC patients with IIPs. However, no study has examined the safety and efficacy of PTX in relapsed SCLC patients with IIPs. Therefore, we conducted a retrospective analyses to evaluate the safety and efficacy of PTX in relapsed SCLC patients with IIPs.

\section{Materials and and methods}

Study population and design. Between January 2010 and August 2017, we enrolled 32 patients (all Japanese) who were diagnosed with both SCLC and IIPs and received first-line chemotherapy at Tokushima University hospital. Of the 32 patients, three died during first-line chemotherapy: Two died from tumor-progression and another died from respiratory infection following febrile neutropenia. They were excluded from the analysis. We retrospectively analyzed clinical features including age, sex, smoking history, performance status (PS), and clinical stage at the end of first-line chemotherapy. Radiologic features of IIPs on high-resolution computed tomography (HRCT) and respiratory function test at the diagnosis of SCLC were also analyzed. The clinical stage was determined on the basis of the international TNM criteria for cancer staging. PS was assessed according to the Eastern Cooperative Oncology Group (ECOG) classification. The cumulative cigarette exposure (pack-years) was calculated by multiplying the average number of packs of cigarettes smoked per day by the number of years for smoking. The diagnosis of IIPs was made according to the reported criteria (22). IIPs were classified into two groups: Idiopathic pulmonary fibrosis (IPF), which consists of usual interstitial pneumonia (UIP) or a possible UIP pattern by HRCT, and non-IPF, which consists of an inconsistent UIP pattern by HRCT according to the official ATS/ERS/JRS/ALAT statement (23). AE of IIPs was diagnosed using the international working group report (24).
Table I. Comparison of the characteristics of SCLC patients with IIPs.

\begin{tabular}{lccc}
\hline Variables & $\begin{array}{c}\text { PTX } \\
n=17\end{array}$ & $\begin{array}{c}\text { BSC } \\
n=12\end{array}$ & P-value \\
\hline Age (years) & & & $0.365^{\mathrm{a}}$ \\
Mean (SEM) & $72.0 \pm 2.3$ & $74.8 \pm 1.3$ & \\
Sex & & & $1.000^{\mathrm{b}}$ \\
Male & $16(94 \%)$ & $11(92 \%)$ & \\
Female & $1(6 \%)$ & $1(8 \%)$ & \\
ECOG PS & & & $0.119^{\mathrm{b}}$ \\
0,1 & $13(76 \%)$ & $5(42 \%)$ & \\
$\geq 2$ & $4(24 \%)$ & $7(58 \%)$ & \\
Clinical stage & & & $0.422^{\mathrm{b}}$ \\
LD & $4(24 \%)$ & $5(42 \%)$ & \\
ED & $13(76 \%)$ & $7(58 \%)$ & \\
Cigarette exposing & & & $0.477^{\circ}$
\end{tabular}

Cigarette exposure (pack-years)

Mean (SEM)

$68.8 \pm 10.280 .3 \pm 12.2$

Type of IIPs

IPF $\quad 6(35 \%) \quad 5(42 \%)$

Non-IPF

$11(65 \%) 7(58 \%)$

Response to first-line therapy

CR, PR

SD, PD

$9(75 \%)$

Type of relapse

Sensitive $\quad 1(6 \%) \quad 3(25 \%)$

Refractory $\quad 16(94 \%) \quad 9(75 \%)$

$1.000^{\mathrm{b}}$

$1.000^{\mathrm{b}}$

$0.279^{\mathrm{b}}$

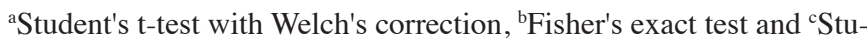
dent's t-test. SCLC, small cell lung cancer; IIPs, idiopathic interstitial pneumonias; PTX, paclitaxel; BSC, best supportive care; SEM, standard error of mean; ECOG PS, the Eastern Cooperative Oncology Group performance status; LD, limited disease; ED, extensive disease; IPF, idiopathic pulmonary fibrosis; CR, complete response; $\mathrm{PR}$, partial response; $\mathrm{SD}$, stable disease; $\mathrm{PD}$, progressive disease.

Statistical analysis. All comparisons between populations were performed by the Fisher's exact test or Student's t-test, as appropriate. ORR was defined using Response Evaluation Criteria in Solid Tumors (25). Disease control rate (DCR) was defined as the proportion of patients with complete response, partial response, and stable disease. Overall survival (OS) was defined as the time from the date of initiation of PTX-containing regimens until the date of death from any cause. PFS was defined as the time from the date of the initiation of PTX-containing regimens until the date of death or evidence of tumor progression. Patients who were alive or no evidence of tumor progression at the time of analysis were censored at the last known date of follow-up. OS and PFS were estimated using the Kaplan-Meier method, and the log-rank test was used to assess differences between groups. Results are reported as the mean \pm standard error of mean (SEM). Adverse events were graded using National Cancer Institute Common Terminology Criteria for Adverse Events, version 4.0. P-values less than 0.05 were considered significant. Statistical analyses 
Table II. Response of second-line chemotherapy for SCLC patients with IIPs.

\begin{tabular}{lrll}
\hline Regimen & $n$ & ORR $n(\%)$ & DCR $n(\%)$ \\
\hline PTX & 12 & $3(25.0)$ & $6(50.0)$ \\
nab-PTX & 4 & $2(50.0)$ & $2(50.0)$ \\
CBDCA+PTX & 1 & $0(0)$ & $0(0)$ \\
All & 17 & $5(29.4)$ & $8(47.1)$ \\
\hline
\end{tabular}

SCLC, small cell lung cancer; IIPs, idiopathic interstitial pneumonias; ORR, overall response rate; DCR, disease control rate; PTX, paclitaxel; nab-PTX, nanoparticle albumin-bound paclitaxel; CBDCA, carboplatin.

Table III. PTX-related adverse events in SCLC patients with IIPs.

\begin{tabular}{lr}
\hline Adverse events & $n(\%)$ \\
\hline Grade $\geq 3$ & \\
Hematologic & $7(47.1)$ \\
Leukopenia & $12(70.6)$ \\
Neutropenia & $2(11.8)$ \\
Anemia & $2(11.8)$ \\
Thrombocytopenia & $2(11.8)$ \\
Febrile neutropenia & \\
Any grade & \\
Non-hematologic & $4(23.5)$ \\
Neuropathy & $5(29.4)$ \\
Pneumonitis & \\
\hline
\end{tabular}

PTX, paclitaxel; SCLC, small-cell lung cancer; IIPs, idiopathic interstitial pneumonias.

were performed using GraphPad PRISM (5.01; GraphPad Software, Inc., La Jolla, CA, USA).

\section{Results}

Characteristics of SCLC patients with IIPs. We identified 29 SCLC patients with IIPs who received first-line chemotherapy of a platinum-based drug plus etoposide. Of the 29 patients, 17 received PTX-containing regimens (PTX group) and 12 received best supportive care (BSC: BSC group) after disease progression. AE of IIPs related to first-line chemotherapy occurred in one patient in the BSC group. Patients in the BSC group had poor PS compared to those in the PTX group, and the ORR to first-line chemotherapy was similar between the groups (Table I). Of the 17 patients that received PTX, all but one were refractory-relapsed cases; 12 received PTX monotherapy, four received nanoparticle albumin-bound PTX monotherapy, and one received combination therapy with carboplatin plus PTX (Tables I and II).

Efficacy of PTX in SCLC patients with IIPs. During the observation period, 14 patients $(82.4 \%)$ in the PTX group

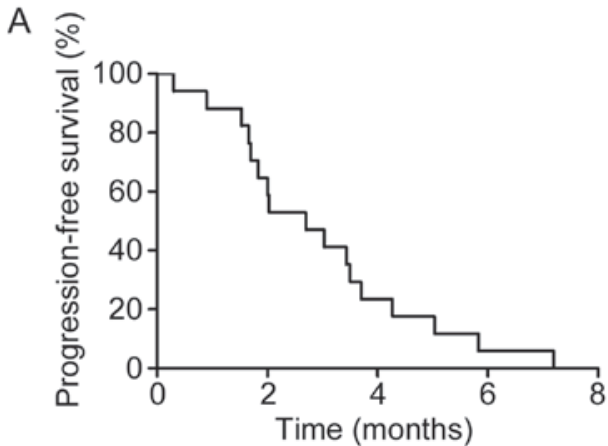

B

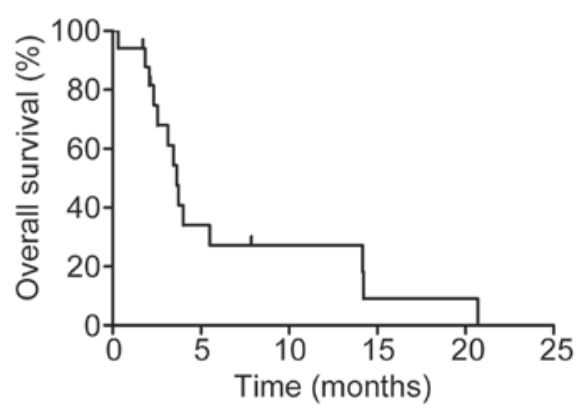

Figure 1. Kaplan-Meier estimates of the probability of progression-free survival and overall survival of PTX for SCLC patients with IIPs. (A) Progression-free survival of PTX for SCLC patients with IIPs (median 2.7 months; 95\% CI, 1.6-3.6 months). (B) overall survival of PTX for SCLC patients with IIPs (median 3.6 months; 95\% CI, 2.3-14.0 months). PTX, paclitaxel; SCLC, small-cell lung cancer; IIPs, idiopathic interstitial pneumonias; CI, confidence interval.

and $10(83.3 \%)$ patients in the BSC group died. The ORR and the DCR of PTX-containing regimens were 29.4 and $47.1 \%$, respectively (Table II). The median PFS and the MST of PTX-containing regimens were 2.7 months [95\% confidence interval (CI), 1.6-3.6 months] and 3.6 months (95\% CI, 2.3-14.0 months), respectively (Fig. 1).

Toxicity of PTX in SCLC patients with IIPs. The median number of PTX treatment cycles was two (range 1-7). The most common grade 3-4 adverse events were hematological toxicities in $12(70.6 \%)$ and seven $(47.1 \%)$ patients with neutropenia and leukopenia, respectively (Table III). Two patients experienced febrile neutropenia (11.8\%) and one of them died from respiratory infection. For non-hematologic toxicity, sensory neuropathy of grade 2 or lower and AE of IIPs were observed in four $(23.5 \%)$ and five patients $(29.4 \%)$, respectively. The characteristics of five patients that experienced AE of IIPs are summarized in Table IV. All received broad-spectrum antibiotics and high-dose intravenous methylprednisolone pulse therapy followed by oral prednisolone. Four recovered from AE of IIPs, but one died from respiratory failure. No significant factor associated with PTX-induced AE of IIPs was observed (Table V). During the observation period, AE of IIPs was not observed in the BSC group.

\section{Discussion}

In this study, we evaluated the safety and efficacy of PTX in relapsed SCLC patients with IIPs and showed that PTX-containing regimens had promising anti-tumor activity 
Table IV. The characteristics of patients who developed PTX-induced AE of IIPs.

\begin{tabular}{lccccc}
\hline & \multicolumn{5}{c}{ Case } \\
\cline { 2 - 6 } Variables & 1 & 2 & 3 & 4 & 5 \\
\hline Age (years) & 67 & 68 & 69 & 69 & 84 \\
Sex & Male & Male & Male & Male & Female \\
ECOG PS & 2 & 0 & 1 & 1 & 1 \\
Clinical stage & IVB & IVB & IVB & IVB & IVA \\
Cigarette exposure (pack-years) & 45 & 64 & 37 & 84 & 20 \\
Type of IIPs & Inconsistent & Inconsistent & UIP & UIP & Inconsistent \\
History of thoracic radiotherapy & No & No & No & No & No \\
KL-6 (U/ml) & 520 & 2,210 & 387 & 2,107 & 377 \\
LDH (IU/l) & 638 & 228 & 346 & 256 & 221 \\
CRP (mg/dl) & 7.9 & 0.4 & 1.5 & 0.3 & 0.2 \\
\%VC (\%) & 104.0 & 86.3 & 87.9 & 94.0 & 56.6 \\
CTCAE Grade & 5 & 3 & 3 & 3 & 3 \\
OS (months) & 0.3 & $2.1^{\text {a }}$ & 3.4 & 3.6 & 4.0 \\
\hline
\end{tabular}

aSurvivor at the end of follow-up. PTX, paclitaxel; AE, acute exacerbation; IIPs, idiopathic interstitial pneumonias; ECOG PS, the Eastern Cooperative Oncology Group performance status; UIP, usual interstitial pneumonias; KL-6, Krebs von den Lungen-6; LDH, lactate dehydrogenase; CRP, C-reactive protein; VC, vital capacity; CTCAE, Common Terminology Criteria for Adverse Events; OS, overall survival.

against refractory-relapsed SCLC patients with IIPs. However, the survival benefit of PTX in relapsed SCLC patients with IIPs appeared to be limited.

Two phase II studies evaluated the effectiveness of PTX for previously treated SCLC patients without IIPs $(19,20)$, and the ORRs of PTX were 20.0 and 29.2\% (19,20). Furthermore, the ORR of PTX with or without carboplatin for both sensitive (39\%) and refractory (61\%) SCLC patients with IIPs in the second line setting was $27.8 \%$ (16). We found that the ORR of PTX-containing regimens in refractory-relapsed SCLC patients with IIPs was $29.4 \%$, which was comparable to previous reports. Because the ORR of second line chemotherapy for refractory-relapsed SCLC patients was $14.8 \%$, PTX may be effective for refractory-relapsed SCLC patients with or without IIPs (4). However, we found that the MST of second-line PTX in refractory-relapsed SCLC patients with IIPs was 3.6 months. The MST of PTX in both sensitive (52\%) and refractory (48\%) relapsed SCLC patients without IIPs was 5.8 months (20). Furthermore, the MST of second-line chemotherapy including PTX in both sensitive (39\%) and refractory (61\%) SCLC patients with IIPs was 7.1 months (16). Compared to previous reports, the survival benefit of PTX in this study was limited, which may be because all patients except for one were refractory-relapsed cases.

Severe toxicities related to PTX may also limit the survival benefit of PTX. Grade 3 or higher pneumonitis related to PTX-induced AE of IIPs was observed in five patients (29.4\%) and one of them died from respiratory failure in spite of high-dose corticosteroid therapy. AE of IIPs related to second-line PTX was observed in $11 \%$ (2/18 patients) of SCLC patients with IPF and at least one of two patients died from pneumonitis (16). In NSCLC patients with IIPs, AE of IIPs related to PTX administered as first-line chemotherapy was observed in $9.5 \%$ (10/105 patients), and the incidence of
AE of IIPs related to PTX was lower than that related to other agents $(21,26-28)$. Therefore, PTX in combination with carboplatin is a good candidate regimen for NSCLC patients with IIPs. An increased risk of AE of IIPs was observed in lung cancer patients with IIPs that received second- or more-line chemotherapy compared to those that received first-line chemotherapy $(13,29)$. Therefore, the risk of AE of IIPs related to PTX in relapsed SCLC patients may be higher compared to that in NSCLC patients that received PTX as a first-line chemotherapy. Although, we did not find any factors associated with PTX-induced AE of IIPs, UIP pattern was reported to be an independent risk factor for chemotherapy-related $\mathrm{AE}$ of interstitial lung disease (ILD) (30). In addition, the baseline serum Krebs von den Lungen-6 (KL-6) level with cut-off values of $1300 \mathrm{U} / \mathrm{ml}$ and a disease severity based on the partial pressure of arterial oxygen $\left(\mathrm{PaO}_{2}\right)$ were associated with the development of AE of IPF $(31,32)$. Consistent with a previous study, baseline serum KL-6 levels were higher in patients with AE of IIPs than those without AE of IIPs, and all but two patients with baseline serum KL-6 levels above $1300 \mathrm{U} / \mathrm{ml}$ developed AE of IIPs. The GAP index helps predicts mortality in patients with IPF (33). A recent study demonstrated that modified GAP index score, which was calculated by four predictors: ILD subtype, sex, age, and forced vital capacity, predicted the risk of AE of ILD associated with chemotherapy in NSCLC patients with ILD (34). The 1-year incidence of AE of ILD in patients with modified GAP index stage I was $14 \%$, and an increased risk of AE of ILD was observed in patients with modified GAP stage II and III. The incidence rate in patients with GAP index stage I was equivalent to that observed in the natural course of IPF patients, and therefore patients with modified GAP index stage I may be indicated for chemotherapy against lung cancer (34). In this study, all five patients who experienced AE of IIPs associated with PTX corresponded to modified GAP 
Table V. Comparison of characteristics between SCLC patients with or without PTX-induced AE of IIPs.

\begin{tabular}{lccc}
\hline & $\begin{array}{c}\text { Without AE of IIPs } \\
n=12\end{array}$ & $\begin{array}{c}\text { With AE of IIPs } \\
n=5\end{array}$ & P-value \\
\hline Agriables & & & $0.779^{\mathrm{a}}$ \\
Mean (SEM) & $72.5 \pm 3.1$ & $71.0 \pm 3.3$ & \\
Sex & & & $0.294^{\mathrm{b}}$ \\
Male & $12(100 \%)$ & $1(20 \%)$ & \\
Female & 0 & $4(80 \%)$ & \\
ECOG PS & & & $0.538^{\mathrm{b}}$ \\
0,1 & $10(83 \%)$ & $2(40 \%)$ & \\
$\geq 2$ & $2(17 \%)$ & & $1.000^{\mathrm{b}}$ \\
Type of IIPs & & $3(60 \%)$ & \\
IPF & $4(33 \%)$ & $2(40 \%)$ & \\
Non-IPF & $8(67 \%)$ & $1120.0 \pm 424.9$ & $0.217^{\mathrm{c}}$ \\
KL-6 (U/ml) & $484.7 \pm 87.5$ & $337.8 \pm 78.3$ & $0.422^{\mathrm{c}}$ \\
LDH (IU/l) & $265.3 \pm 21.5$ & $2.1 \pm 1.5$ & $0.758^{\mathrm{a}}$ \\
CRP (mg/dl) & $2.7 \pm 1.2$ & $85.8 \pm 7.9$ & $0.708^{\mathrm{a}}$ \\
\%VC (\%) & $80.5 \pm 8.8$ & & \\
\hline
\end{tabular}

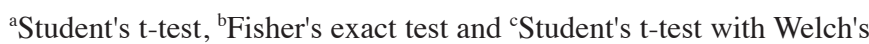
correction. SCLC, small cell lung cancer; PTX, paclitaxel; AE, acute exacerbation; IIPs, idiopathic interstitial pneumonias; SEM, standard error of mean; ECOG PS, the Eastern Cooperative Oncology Group performance status; IPF, idiopathic pulmonary fibrosis; KL-6, Krebs von den Lungen-6; LDH, lactate dehydrogenase; CRP, C-reactive protein; VC, vital capacity.

stage II (data not shown). The indication of PTX in relapsed SCLC patients with IIPs should be carefully considered, especially in those with UIP pattern, high baseline serum KL-6 level, low baseline $\mathrm{PaO}_{2}$, and modified GAP stage II or III.

In this study, grade 3 or 4 neutropenia was observed in 12 (70.6\%) of 17 patients. The high incidence of neutropenia was comparable to past clinical trials of weekly PTX or topotecan for relapsed SCLC patients $(6,20)$. Although most adverse events associated with myelosuppression were manageable, one patient died from respiratory infection caused from febrile neutropenia. A clinical trial of weekly PTX for relapsed SCLC patients also reported one case $(4.8 \%)$ of treatment-related death caused by neutropenic pneumonia (20). When we administer PTX-containing regimens in relapsed SCLC patients, caution is warranted for neutropenia and infection associated with neutropenia.

There were several limitations in the present study. First, this study did not have sufficient power to evaluate the precise clinical benefit of PTX and may have overestimated the incidence of PTX-related AE of IIPs because of the small number of patients and the retrospective nature of the study at a single institution. Second, the diagnoses of IIPs were made clinically with no histological confirmation in all patients. Because SCLC patients usually have no indications for surgery at diagnosis and should receive chemotherapy as soon as possible because of its rapid growth, it is usually difficult to obtain a specimen from non-tumor-bearing fibrotic areas.
In conclusion, this was the first retrospective study to evaluate the safety and efficacy of PTX-containing regimens in relapsed SCLC patients with IIPs. Although PTX-containing regimens demonstrated promising anti-tumor activity against relapsed SCLC with IIPs, the survival benefit was limited because of the high incidence of PTX-related AE of IIPs and treatment-related death. The administration of PTX in relapsed SCLC patients with IIPs should carefully considered and be performed with particular care because of pulmonary toxicity.

\section{Ackonowledgements}

Not applicapable.

\section{Funding}

No funding was received.

\section{Availability of data and materials}

All data generated or analyzed during this study are included in this published article.

\section{Authors' contributions}

AS, MH and YN designed the study. AS, HO and KO contributed to the data acquisition. AS, MH, HG and $\mathrm{HN}$ analyzed and interpreted the patient data. AS, MH and YN drafted the manuscript. All authors read and approved the final manuscript.

\section{Ethics approval and consent to participate}

The study was approved by the Institutional Review Board of Tokushima University Hospital (IRB approval number: 2973). For this type of study formal consent was not required. Information about the current study was disclosed to patients instead of obtaining their written informed consent, and patients who declined to participate were excluded.

\section{Patient consent for publication}

Not applicable.

\section{Competing interests}

The authors declare that they have no competing interests.

\section{References}

1. Gazdar AF, Bunn PA and Minna JD: Small-cell lung cancer: What we know, what we need to know and the path forward. Nat Rev Cancer 17: 725-737, 2017.

2. Früh M, De Ruysscher D, Popat S, Crinò L, Peters S, Felip E and ESMO Guidelines Working Group: Small-cell lung cancer (SCLC): ESMO clinical practice guidelines for diagnosis, treatment and follow-up. Ann Oncol 6 (24 Suppl): vi99-vi105, 2013.

3. Kalemkerian GP, Loo BW, Akerley W, Attia A, Bassetti M, Boumber Y, Decker R, Dobelbower MC, Dowlati A, Downey RJ, et al: NCCN guidelines insights: Small cell lung cancer, Version 2.2018. J Natl Compr Cancer Netw 16: 1171-1182, 2018. 
4. Owonikoko TK, Behera M, Chen Z, Bhimani C, Curran WJ, Khuri FR and Ramalingam SS: A systematic analysis of efficacy of second-line chemotherapy in sensitive and refractory small-cell lung cancer. J Thorac Oncol 7: 866-872, 2012.

5. O'Brien ME, Ciuleanu TE, Tsekov H, Shparyk Y, Čučeviá B, Juhasz G, Thatcher N, Ross GA, Dane GC and Crofts T: Phase III trial comparing supportive care alone with supportive care with oral topotecan in patients with relapsed small-cell lung cancer. J Clin Oncol 24: 5441-5447, 2006.

6. Eckardt JR, von Pawel J, Pujol JL, Papai Z, Quoix E, Ardizzoni A, Poulin R, Preston AJ, Dane G and Ross G: Phase III study of oral compared with intravenous topotecan as second-line therapy in small-cell lung cancer. J Clin Oncol 25: 2086-2092, 2007.

7. Von Pawel J, Jotte R, Spigel DR, O'Brien ME, Socinski MA, Mezger J, Steins M, Bosquee L, Bubis J, Nackaerts K, et al: Randomized phase III trial of Amrubicin versus topotecan as second-line treatment for patients with small-cell lung cancer. J Clin Oncol 32: 4012-4019, 2014.

8. Antonia SJ, López-Martin JA, Bendell J, Ott PA, Taylor M, Eder JP, Jäger D, Pietanza MC, Le DT, de Braud F, et al: Nivolumab alone and nivolumab plus ipilimumab in recurrent small-cell lung cancer (CheckMate 032): A multicentre, open-label, phase 1/2 trial. Lancet Oncol 17: 883-895, 2016.

9. Sato T, Teramukai S, Kondo H, Watanabe A, Ebina M, Kishi K, Fujii Y, Mitsudomi T, Yoshimura M, Maniwa T, et al: Impact and predictors of acute exacerbation of interstitial lung diseases after pulmonary resection for lung cancer. J Thorac Cardiovasc Surg 147: 1604-1611.e3, 2014.

10. Raghu G, Nyberg F and Morgan G: The epidemiology of interstitial lung disease and its association with lung cancer. $\mathrm{Br} \mathrm{J}$ Cancer 2 (Suppl 91): S3-S10, 2004.

11. Togashi Y, Masago K, Handa T, Tanizawa K, Okuda C, Sakamori Y, Nagai H, Kim YH and Mishima M: Prognostic significance of preexisting interstitial lung disease in Japanese patients with small-cell lung cancer. Clin Lung Cancer 13: 304-311, 2012.

12. Minegishi Y, Kuribayashi H, Kitamura K, Mizutani H, Kosaihira S, Okano T, Seike M, Azuma A, Yoshimura A, Kudoh S and Gemma A: The feasibility study of carboplatin plus etoposide for advanced small cell lung cancer with idiopathic interstitial pneumonias. J Thorac Oncol 6: 801-807, 2011.

13. Yoshida T, Yoh K, Goto K, Niho S, Umemura S, Ohmatsu H and Ohe Y: Safety and efficacy of platinum agents plus etoposide for patients with small cell lung cancer with interstitial lung disease. Anticancer Res 33: 1175-1180, 2013.

14. Watanabe N, Taniguchi H, Kondoh Y, Kimura T, Kataoka K, Nishiyama O, Kondo M and Hasegawa Y: Chemotherapy for extensive-stage small-cell lung cancer with idiopathic pulmonary fibrosis. Int J Clin Oncol 19: 260-265, 2014

15. Pelayo Alvarez M, Westeel V, Cortés-Jofré M and Bonfill Cosp X: Chemotherapy versus best supportive care for extensive small cell lung cancer. Cochrane Database Syst Rev 27: CD001990, 2013.

16. Fujimoto D, Shimizu R, Kato R, Sato Y, Kogo M, Ito J, Teraoka S, Otoshi T, Nagata K, Nakagawa A, et al: Second-line chemotherapy for patients with small cell lung cancer and interstitial lung disease. Anticancer Res 35: 6261-6266, 2015.

17. Enomoto $\mathrm{Y}$, Inui $\mathrm{N}$, Imokawa $\mathrm{S}$, Karayama M, Hasegawa $\mathrm{H}$, Ozawa Y, Matsui T, Yokomura K and Suda T: Safety of topotecan monotherapy for relapsed small cell lung cancer patients with pre-existing interstitial lung disease. Cancer Chemother Pharmacol 76: 499-505, 2015.

18. Miura Y, Saito Y, Atsumi K, Takeuchi S, Miyanaga A, Mizutani H, Minegishi Y, Noro R, Seike M, Shinobu K, et al: Interstitial lung disease associated with amrubicin chemotherapy in patients with lung cancer: A single institutional study. Jpn J Clin Oncol 46: 674-680, 2016.

19. Smit EF, Fokkema E, Biesma B, Groen HJ, Snoek W and Postmus PE: A phase II study of paclitaxel in heavily pretreated patients with small-cell lung cancer. Br J Cancer 77: 347-351, 1998.

20. Yamamoto N, Tsurutani J, Yoshimura N, Asai G, Moriyama A, Nakagawa K, Kudoh S, Takada M, Minato Y and Fukuoka M: Phase II study of weekly paclitaxel for relapsed and refractory small cell lung cancer. Anticancer Res 26: 777-781, 2006.
21. Minegishi Y, Sudoh J, Kuribayasi H, Mizutani H, Seike M, Azuma A, Yoshimura A, Kudoh S and Gemma A: The safety and efficacy of weekly paclitaxel in combination with carboplatin for advanced non-small cell lung cancer with idiopathic interstitial pneumonias. Lung Cancer 71: 70-74, 2011.

22. Travis WD, Costabel U, Hansell DM, King TE Jr, Lynch DA, Nicholson AG, Ryerson CJ, Ryu JH, Selman M, Wells AU, et al: An official American Thoracic Society/European Respiratory Society statement: Update of the international multidisciplinary classification of the idiopathic interstitial pneumonias. Am J Respir Crit Care Med 188: 733-748, 2013.

23. Raghu G, Collard HR, Egan JJ, Martinez FJ, Behr J, Brown KK, Colby TV, Cordier JF, Flaherty KR, Lasky JA, et al: An official ATS/ERS/JRS/ALAT statement: Idiopathic pulmonary fibrosis: Evidence-based guidelines for diagnosis and management. Am J Respir Crit Care Med 183: 788-824, 2011.

24. Collard HR, Ryerson CJ, Corte TJ, Jenkins G, Kondoh Y, Lederer DJ, Lee JS, Maher TM, Wells AU, Antoniou KM, et al: Acute exacerbation of idiopathic pulmonary fibrosis an international working group report. Am J Respir Crit Care Med 194: 265-275, 2016

25. Eisenhauer EA, Therasse P, Bogaerts J, Schwartz LH, Sargent D, Ford R, Dancey J, Arbuck S, Gwyther S, Mooney M, et al: New response evaluation criteria in solid tumours: Revised RECIST guideline (version 1.1). Eur J Cancer 45: 228-247, 2009.

26. Kenmotsu H, Naito T, Mori K, Ko R, Ono A, Wakuda K, Imai H, Taira T, Murakami H, Endo M and Takahashi T: Effect of platinum-based chemotherapy for non-small cell lung cancer patients with interstitial lung disease. Cancer Chemother Pharmacol 75: 521-526, 2015.

27. Shimizu R, Fujimoto D, Kato R, Otoshi T, Kawamura T, Tamai K, Matsumoto T, Nagata K, Otsuka K, Nakagawa A, et al: The safety and efficacy of paclitaxel and carboplatin with or without bevacizumab for treating patients with advanced nonsquamous non-small cell lung cancer with interstitial lung disease. Cancer Chemother Pharmacol 74: 1159-1166, 2014.

28. Shukuya T, Ishiwata T, Hara M, Muraki K, Shibayama R, Koyama R and Takahashi K: Carboplatin plus weekly paclitaxel treatment in non-small cell lung cancer patients with interstitial lung disease. Anticancer Res 30: 4357-4361, 2010.

29. Kakiuchi S, Hanibuchi M, Tezuka T, Saijo A, Otsuka K, Sakaguchi S, Toyoda Y, Goto H, Kawano H, Azuma M, et al: Analysis of acute exacerbation of interstitial lung disease associated with chemotherapy in patients with lung cancer: A feasibility of S-1. Respir Investig 55: 145-152, 2017.

30. Kenmotsu H, Naito T, Kimura M,Ono A, Shukuya T, Nakamura Y, Tsuya A, Kaira K, Murakami H, Takahashi T, et al: The risk of cytotoxic chemotherapy-related exacerbation of interstitial lung disease with lung cancer. J Thorac Oncol 6: 1242-1246, 2011

31. Ohshimo S, Ishikawa N, Horimasu Y, Hattori N, Hirohashi N, Tanigawa K, Kohno N, Bonella F, Guzman J and Costabel U: Baseline KL-6 predicts increased risk for acute exacerbation of idiopathic pulmonary fibrosis. Respir Med 108: 1031-1039, 2014.

32. Homma S, Sugino K and Sakamoto S: The usefulness of a disease severity staging classification system for IPF in Japan: 20 years of experience from empirical evidence to randomized control trial enrollment. Respir Investig 53: 7-12, 2015.

33. Ley B, Ryerson CJ, Vittinghoff E, Ryu JH, Tomassetti S, Lee JS, Poletti V, Buccioli M, Elicker BM, Jones KD, et al: A multidimensional index and staging system for idiopathic pulmonary fibrosis. Ann Intern Med 156: 684-691, 2012.

34. Kobayashi H, Naito T, Omae K, Omori S, Nakashima K, Wakuda K, Ono A, Kenmotsu H, Murakami H, Endo M and Takahashi T: ILD-NSCLC-GAP index scoring and staging system for patients with non-small cell lung cancer and interstitial lung disease. Lung Cancer 121: 48-53, 2018. 